\title{
PRÁCTICAS DE MENTORÍA PARA LA INDUCCIÓN DE DOCENTES PRINCIPIANTES: ANÁLISIS DE CUATRO CASOS CHILENOS AL INICIO DEL PROCESO
}

\author{
Solange Gorichon ${ }^{1}$, Macarena Salas², María José Araos ${ }^{3}$, \\ Mariluz Yáñez ${ }^{4}$, Andrés Rojas-Murphy ${ }^{5}$, Geraldine \\ Jara-Chandía ${ }^{6}$
}

\begin{abstract}
RESUMEN
La presente investigación busca profundizar en las prácticas de mentoría que se utilizan, en una fase inicial de este proceso en Chile, para acompañar, apoyar y facilitar la inserción profesional de docentes principiantes en sus respectivas comunidades educativas. Se propone un enfoque cualitativo, de carácter exploratorio y descriptivo interpretativo, que considera el estudio de cuatro casos, conformados por cuatro duplas de mentores y principiantes, con el fin de realizar una indagación detallada y profunda de este acompañamiento docente. Para esto se caracterizan las reflexiones que se promueven, los saberes que se movilizan y las estrategias que utilizan los docentes mentores durante el proceso de mentoría. Los hallazgos evidencian la mediación de elementos contextuales y relacionales vinculados con la organización del proceso de mentoría. Asimismo, se identifica la preeminencia de focos temáticos sobre los cuales se conversa y algunos marcos interpretativos, saberes y recursos que moviliza el mentor durante el acompañamiento. Este estudio pretende contribuir al Sistema Nacional de Inducción, que emerge recientemente en Chile, con la generación de recomendaciones que contribuyan a mejorar la formación de mentores, a fortalecer el proceso de mentoría y a generar orientaciones y propuestas para la política educativa en esta área.
\end{abstract}

Conceptos clave: mentorías, docentes principiantes, mentor, prácticas de mentoría, aprendizaje profesional.

1 Universidad Alberto Hurtado, Santiago, Chile. Contacto: sgoricho@uahurtado.cl

2 Pontificia Universidad Católica de Chile, Santiago, Chile. Contacto: msalas1@uc.cl

3 Investigadora independiente, Santiago, Chile. Contacto: mariajose.araosn@gmail.com

4 Universidad Alberto Hurtado, Santiago, Chile. Contacto: myanez@uahurtado.cl

5 Universidad de Chile, Santiago, Chile. Contacto: andres.rojasmurphy@uchile.cl

6 Universidad San Sebastián, Santiago, Chile. Contacto: geraldine.jara@uss.cl 


\title{
MENTORING PRACTICES FOR THE INSERTION OF NEW TEACHERS: ANALYSIS OF FOUR CHILEAN CASES AT THE BEGINNING OF THE PROCESS
}

\begin{abstract}
This research seeks to deepen understanding of the mentoring practices used, in an initial phase of this process in Chile, to accompany, support and facilitate the professional insertion of novice teachers into their educational communities. The investigation is based on a qualitative and exploratory, descriptive-interpretative approach, that considers the study of four cases, consisting of four pairs of mentors and novice teachers. This strategy permitted a detailed and in-depth investigation of this type of accompaniment, which considered the characterization of the reflection and knowledge promoted as well the strategies used by the mentors during the mentoring process. The findings show the mediation of contextual and relational elements that are linked to the organization of the mentoring process. Likewise, the study identifies the preeminence of certain thematic focuses, frameworks as well as knowledge and resources that the mentor uses during the accompaniment. This study aims to contribute to the National Induction System, which has recently emerged in Chile, by promoting recommendations that will help improve mentor training, strengthen the mentoring process, and generate guidelines and proposals for educational policy in this area.
\end{abstract}

Key concepts: mentoring, novice teachers, mentor, mentoring practices, professional learning. 
14 PRÁCTICAS DE MENTORÍA PARA LA INDUCCIÓN DE DOCENTES PRINCIPIANTES: ANÁLISIS DE CUATRO CASOS CHILENOS AL INICIO DEL PROCESO - S. Gorichon, M. Salas, M. Araos, M. Yáñez, A. Rojas-Murphy, G. Jara-Chandía

\section{Introducción}

Con la promulgación de la Ley 20.903, que crea el Sistema de Desarrollo Profesional Docente, se instala el Sistema Nacional de Inducción y Mentoría como un derecho al que pueden optar todos los profesores principiantes que ingresen al ejercicio profesional en el sistema público. Este derecho considera que el profesor principiante pueda recibir el apoyo de un profesional formado como mentor en universidades, públicas o privadas, que se encuentren acreditadas por Cpeip $^{7}$ para formación de mentores. Entre 2018 y 2022 el Sistema de Inducción funcionará con gradualidad y priorización de postulantes, pero a partir de 2023 se ampliará para todos los interesados e interesadas que ingresen por primera vez a un establecimiento con financiamiento del Estado (Mineduc, 2016).

La formación de mentores, impartida por distintas universidades en convenio con el Cpeip, tiene como objetivo ofrecer experiencias que permitan fortalecer la interacción entre los profesores experimentados y principiantes, a partir de diálogos y reflexiones que tengan como foco el desarrollo de elementos pedagógicos, competencias comunicativas y análisis reflexivo de las propias prácticas.

En el plano internacional, durante los últimos años varias investigaciones han sugerido la necesidad de avanzar en estudios que relacionen los procesos de pensamiento de mentores con evidencia que se refiera a su comportamiento y desempeño (OrlandBarak, 2016). En este sentido, si bien existen estudios recientes que profundizan en las creencias, conocimientos y prácticas de mentores de manera conjunta (Achinstein \& Athanases, 2005; Athanases \& Martin, 2006; Cochran-Smith, 2003; Orland-Barak, 2010), todavía se trata de investigaciones incipientes, que precisan ser enriquecidas a partir de los contextos particulares en que se desarrollan los programas de mentoría. De las investigaciones centradas en la

7 El Centro de Perfeccionamiento, Experimentación e Investigaciones Pedagógicas del Ministerio de Educación (Cpeip) es el organismo encargado de implementar la ley que crea el Sistema de Desarrollo Profesional Docente en Chile. A su vez, define las bases de la política pública que regula y orienta el desarrollo profesional de los docentes en Chile. 
relación entre creencias, conocimientos y prácticas destaca la revisión de literatura de Crutcher y Naseem (2016), que identifica ciertas prácticas de mentoría efectivas, agrupadas en i) reflexiones críticas y retroalimentaciones, ii) procesos de modelaje, iii) colaboración y iv) conocimiento de las necesidades de los principiantes. Asimismo, Billingsley, Bettini y Jones (2019) profundizan en la incidencia que tendrían las high-leverage practices (HLPs) para la inducción de profesores principiantes. En Chile, varios estudios (e.g., Melero, 2017; Solís, Núñez, Vásquez, Contreras y Ritterhaussen, 2016) declaran la necesidad de investigar sobre experiencias concretas de mentoría para profundizar en los alcances de este proceso. En este sentido, la presente investigación busca enriquecer la comprensión de este acompañamiento, a partir de la caracterización de mentorías que aún se encuentran en una fase de implementación en Chile. Más allá de intentar aproximarse a la concepción de "buenas prácticas" de mentoría, se busca profundizar en la descripción respecto de "qué hacen" aquellos docentes que acompañan a los principiantes.

Cabe mencionar que las investigaciones que abordan estas temáticas en el contexto chileno se relacionan con las dificultades de los docentes principiantes (Ávalos, 2009; Ávalos \& Valenzuela, 2016) y con la definición del rol del mentor (Inostroza, Jara y Tagle, 2010). Este estudio pretende caracterizar cómo se efectúa el proceso de mentoría — que se encuentra en su fase inicial— a partir de la consideración de los siguientes objetivos: i) analizar el proceso reflexivo que desarrollan mentores y profesores principiantes durante las sesiones de mentoría en su fase inicial, ii) identificar y analizar los marcos interpretativos y saberes que los mentores relevan y movilizan en las sesiones iniciales, y iii) describir las estrategias que utilizan los mentores para facilitar la inserción profesional de los docentes principiantes en sus respectivas comunidades educativas ${ }^{8}$.

8 Cabe precisar que este estudio no estuvo exento de dificultades, que impidieron llevarlo a cabo como estaba planificado: inicio tardío, en septiembre de 2019, del proceso de mentoría; surgimiento del "estallido social" el 18 de octubre, que provocó irregularidad en el acompañamiento; cambios contractuales de algunos docentes principiantes y consiguiente imposibilidad de continuar participando en el proyecto, y, por último, la pandemia declarada por el COVID 19, que provocó la suspensión de clases a nivel nacional. 
16 PRÁCTICAS DE MENTORÍA PARA LA INDUCCIÓN DE DOCENTES PRINCIPIANTES: ANÁLISIS DE CUATRO CASOS CHILENOS AL INICIO DEL PROCESO - S. Gorichon, M. Salas, M. Araos, M. Yáñez, A. Rojas-Murphy, G. Jara-Chandía

\section{Antecedentes teóricos}

Estudios chilenos (e.g., Cisternas, 2016; Ruffinelli, 2013; 2014; Solís et al., 2016) dan cuenta de las dificultades que enfrentarían los docentes durante los primeros años; algunos de estos elementos se relacionarían con la formación inicial y con los problemas para insertarse en los centros educativos. Estas situaciones permiten relevar la importancia de contar con programas de apoyo durante los primeros años de ejercicio profesional, con el fin de desarrollar competencias y mejorar el nivel de confianza de profesores principiantes a la hora de enfrentar un nuevo escenario laboral.

En respuesta a la necesidad de mejorar la calidad de la educación en Chile, durante 2005 se crea una comisión compuesta por especialistas del mundo académico, quienes proponen lineamientos generales para desarrollar programas de inducción docente. Durante 2006, el Ministerio de Educación, a través del Cpeip, invita a instituciones de educación superior a una licitación para la formulación de propuestas para formar mentores. La Universidad Católica de Temuco genera el primer programa piloto, que luego se replicará en 2008 por la Pontificia Universidad Católica de Valparaíso (Ortúzar, Flores, Milesi, Müller y Ayala, 2011). Estas experiencias dejan en evidencia la necesidad de contar con un marco políticoinstitucional que considere el primer año de inserción como parte de la formación docente, y de generar alianzas con los directores de los establecimientos, de modo de comprometer a la comunidad escolar en la inducción de los principiantes (Nuñez, Pino y López, 2011). En 2009, el Cpeip, ante la solicitud del Mineduc, abre un concurso para formar mentores a partir de la generación de diplomados. Si bien a esta iniciativa se suman diferentes universidades del país, dicho programa se suspende durante 2010 y 2011 por falta de financiamiento. Este sistema de mentoría volverá a emerger, años más tarde, al alero de la promulgación de la Ley 20.903.

Los sistemas de apoyo docente se han focalizado mayoritariamente en la importancia de desarrollar la profesionalización docente. En este sentido, la inserción de los principiantes debiera constituir un proceso comprensivo, coherente, sostenido y 
organizado por la autoridad educativa (Vaillant y Marcelo, 2015). En definitiva, lo que busca es que el principiante pueda convertirse en un experto adaptativo, es decir, capaz de aprender a lo largo de toda su trayectoria profesional con un alto nivel de disposición a cambiar sus competencias continuamente, profundizándolas y ampliándolas a partir de la consideración de necesidades emergentes (Bransford, Derry, Berliner, Hammerness \& Beckett, 2005).

Si bien se ha señalado que los cinco primeros años del ejercicio profesional resultan claves para la adquisición de competencias fundamentales para la práctica; el primer y segundo año son cruciales para definir si el docente principiante permanecerá o abandonará la docencia (Marcelo, 2002). Para Ávalos y Valenzuela (2016), las principales motivaciones que incidirían en las altas tasas de deserción docente durante los primeros años se referirían a: i) insatisfacción con las oportunidades de desarrollo profesional, ii) condiciones laborales precarias asociadas a bajos ingresos económicos, iii) insatisfacción con la gestión directiva, iv) escasa percepción de autonomía profesional y v) escaso apoyo de otros profesores. En concordancia con esto último, Gaete, Navarrete, Pino y Mansilla (2017) insisten en la relevancia del apoyo de otros profesionales para adentrarse e interpretar la cultura escolar en la que se inserta el profesor principiante. Ambas investigaciones sugieren que la deserción estaría vinculada con factores contextuales que habría que considerar para comprender la realidad de los principiantes.

Por su parte, Marcelo (2002) señala que esta inserción profesional se caracterizaría por las dudas, inseguridades y ansiedades que se producirían en los profesores principiantes. Algunos docentes logran enfrentar con éxito los primeros años, adaptándose rápidamente a las culturas escolares en que se insertan; mientras, existe un porcentaje considerable que será superado por los contextos escolares, lo que incide en la decisión de abandonar la profesión (Solís et al., 2016). En esta línea, nuevos elementos permitirían ahondar en los principales desafíos experimentados por los principiantes: i) dificultad con la interiorización de normas institucionales, el trabajo con pares y otros profesionales, la relación profesional con apoderados y el monitoreo de los comportamientos y aprendizajes de los alumnos 
18 PRÁCTICAS DE MENTORÍA PARA LA INDUCCIÓN DE DOCENTES PRINCIPIANTES: ANÁLISIS DE CUATRO CASOS CHILENOS AL INICIO DEL PROCESO - S. Gorichon, M. Salas, M. Araos, M. Yáñez, A. Rojas-Murphy, G. Jara-Chandía

en el aula (Marcelo, 2009); ii) problemas personales de índole económica o familiar; iii) elementos relacionados con la dimensión pedagógica, vinculados con relaciones con alumnos y dominio de los conocimientos que se deben enseñar (Huberman, Gonauer \& Marti, 1993); iv) limitaciones de oportunidades en el aula para realizar innovaciones pedagógicas (Rodríguez, 2007); v) dificultades con el manejo del tiempo, interacciones personales complejas, asumir distintas labores de forma simultánea y generación de estrés producido por la sensación de ser evaluado por otros. Respecto de la planificación y el manejo de la clase, se reconoce la dificultad de trabajar con lineamientos curriculares y el agobio por cubrir todas materias escolares. Por último, en el ámbito de la evaluación, se observa vi) dificultades frente a la escasez de evaluaciones formativas respecto del desafío que significa la construcción de instrumentos evaluativos adecuados para medir lo que efectivamente se enseña al estudiante (Thompson, 2007). En Chile, Cisternas (2016) señala que las dificultades que se presentan al profesor principiante dependen de tres factores: i) oportunidades que la formación inicial proporcionó para problematizar la realidad de la escuela, ii) características de la inserción a la institución escolar y iii) condiciones personales del profesor principiante. En este contexto, es preciso contemplar las dificultades que enfrentan los principiantes considerando la realidad contextual de los establecimientos a los que ingresan. En este sentido, esta investigación pretende abordar el estudio de prácticas situadas que emergen a partir de diferentes contextos escolares.

\subsection{Proceso de mentorías}

La mentoría "tiene por objeto conducir el proceso de inducción al inicio del ejercicio profesional de los docentes principiantes, para la formación de su desarrollo profesional, que fomenta el trabajo colaborativo y la retroalimentación pedagógica" (Mineduc, 2018, p. 1), facilitando con ello la inserción a la docencia y a la comunidad educativa en la cual se inserta. Las mentorías, en este sentido, buscan propiciar relaciones colaborativas entre mentor y principiante, profundizando en el aprendizaje y en el desarrollo profesional de ambos (Lipton \& Wellman, 2005). En específico, el mentor y el docente principiante compartirán el desafío de diseñar, ejecutar y 
evaluar un plan de mentoría, que considere la realización de un conjunto sistemático de actividades, metódicamente organizadas, que potencien en el principiante el desarrollo de su identidad profesional y capacidades reflexivas, la promoción de retroalimentaciones pedagógicas y el desarrollo de sus competencias profesionales en pos de mejorar su desempeño y facilitar su inserción al ejercicio profesional (Mineduc, 2016).

En este punto, el desarrollo de competencias reflexivas resulta relevante, ya que promover la reflexión del profesorado sobre su docencia resulta clave para generar una transformación de la propia práctica. Efectivamente, el ejercicio reflexivo que realizan los docentes sobre su desempeño, a la luz de los saberes didácticos, disciplinares y pedagógicos, puede contribuir a la toma de conciencia de sus propias acciones docentes. Es justamente en estos espacios reflexivos donde los docentes movilizan una serie de conocimientos y construyen un saber pedagógico (Pérez-Gómez, 2010; Sanjurjo, 2012; Tardif, 2004). En este sentido, se trata de lograr que los docentes desarrollen un tipo de saber, de conocimientos y de pensamiento experiencial, que les permita ampliar las interpretaciones de las situaciones que emergen de la práctica, de manera que puedan orientar sus intervenciones educativas considerando el conocimiento teórico adquirido. En el proceso de mentoría, esta reflexión se iniciará con la descripción de algo observado o de algún incidente crítico que permita movilizar ciertas conversaciones. Luego se procederá a profundizar en algo más cercano a la acción, lo que dará paso a un ciclo de revisión, selección, aprendizaje y plan de acción (Iafrancesco, 2003; Malderez \& Bodóczky, 1999; Orland-Barak \& Klein, 2005). En este ejercicio se releva la importancia de las "preguntas reflexivas" planteadas por el mentor, lo que luego, idealmente, debería transformarse en un ejercicio autónomo por parte del profesor principiante.

Respecto de las intervenciones en los procesos de mentoría, Díaz y Bastías (2013) plantean tres tipos: i) directivas, referidas a un discurso de corte prescriptivo, en las que el mentor cumple con un rol similar al del supervisor; ii) alternativas, en las que el mentor sigue manteniendo el control del proceso de enseñanza-aprendizaje, pero propone una serie de alternativas que se adecuan al profesor principiante $y$, por 
último, iii) no directivas, caracterizadas por dar cuenta de interacciones horizontales, democráticas y reflexivas. Ruffinelli (2020), en esta misma línea, propone otros dos tipos: directivo, que se focaliza en la transmisión y reproducción de la información, en la que predomina la noción de que antes de la práctica debe adquirirse la teoría, y constructivista, que asume una mirada compleja, comprensiva, multidimensional y situada del proceso de enseñanza-aprendizaje, considerando que la instancia de reflexión y análisis de un saber práctico se construye a partir de la experiencia y de los saberes profesionales. A partir de esta reflexión será posible reconfigurar los saberes disponibles y generar nuevo conocimiento profesional.

Según Hirmas y Cortés (2015), en los procesos reflexivos tradicionales ha predominado el enfoque directivo, anclado en la persistencia de prácticas tradicionales, a pesar de la emergencia de nuevos discursos (Russell, 2012). La preeminencia de un enfoque más o menos directivo tenderá a movilizar determinados saberes. Por ejemplo, al generarse una interacción y reflexión más directiva, quien reflexione tendrá la oportunidad de movilizar creencias y saberes que le permitan conectar teoría y práctica en pos de poder analizar los contextos pedagógicos (Jaeger, 2013). Por otra parte, al predominar una discusión menos directiva se abrirán espacios para construir representaciones propias de la enseñanza y explorar nuevas vías de reflexión (Moore-Russo \& Wilsey, 2014).

\subsection{Roles y características de los mentores}

Durante el proceso de inducción, el mentor necesitará desplegar una serie de actividades y apoyos destinados al profesor principiante, que se relacionarán con la preparación de clases, la implementación para el desarrollo de habilidades de todos los estudiantes, la evaluación y retroalimentación para mejorar el proceso de enseñanza-aprendizaje, el análisis de prácticas pedagógicas efectivas en el aula y los resultados de aprendizaje de los estudiantes. Esto implica que el mentor contará con una serie de capacidades relacionadas con la observación de la práctica pedagógica; la reflexión crítica sobre dichas prácticas para fortalecer la autonomía del principiante; el establecimiento de una relación dialógica clara que posibilite la reflexión; el monitoreo y 
la retroalimentación del desarrollo de estrategias pedagógicas para propiciar aprendizajes en los estudiantes, y el manejo de información del sistema educativo, políticas actuales y reglamentos que sean relevantes para el desempeño del docente principiante (Cpeip, s/f).

Estas capacidades resultan indispensables en la configuración de los diferentes roles que pueda adoptar el mentor para el desarrollo profesional de los principiantes y para sus procesos de inserción profesional (Marcelo, 2002). Por esto, Malderez y Wedell (2007) enfatizan en determinados roles que debiera cumplir un mentor: i) Acculturator, ayuda a los principiantes a ser conscientes y comprender la cultura de la institución educacional en la que se insertan, ii) Model, modela actitudes positivas que influyen en las formas en que los principiantes se aproximan al aprendizaje y se comprometen con su desarrollo profesional, iii) Support, construye una relación de confianza y cercanía con los principiantes, mostrando compromiso con su desarrollo personal y profesional, iv) Sponsor, apoya a los principiantes para ser aceptados en la comunidad profesional de la institución educacional en la que se están insertando, y v) Educator, media o genera los andamiajes para que los principiantes logren construir sus aprendizajes.

En este punto, todos los roles mencionados consideran la importancia de que el mentor sea capaz de modelar una "práctica reflexiva" para el principiante, mediante la cual pueda visualizar al mentor como un profesional poseedor de una visión crítica y reflexiva. Esto le ayudará a concebir su práctica como socialmente contextualizada, pudiendo afrontar la complejidad y la diversidad, presentes en el aula, desde una reflexión profunda sobre la enseñanza.

\subsection{Factores que influyen en el desarrollo de las mentorías}

El Mineduc (2016) reconoce que la docencia se realiza y consolida en el trabajo colaborativo; por esto, resulta relevante considerar el espacio físico y el tiempo considerado para dialogar, analizar y tomar decisiones en conjunto. En este sentido, es importante precisar que las mentorías no corresponden a una supervisión evaluativa, sino más bien a una instancia de desarrollo profesional. 
Por su parte, Malderez y Wedell (2007) enfatizan en la importancia de que el mentor pueda apoyar el aprendizaje del principiante a partir de la escucha y la realización de conversaciones informadas y estructuradas. Asimismo, resulta relevante considerar los aspectos socioemocionales que mediarán la relación entre mentor y docente principiante. Aquí la escucha activa podría evidenciar alguna intención subyacente o bien una actitud de calidez y apoyo generados por el mentor, como antesala a la implementación de una serie de estrategias (Díaz y Bastías, 2013), de modo que el acompañamiento de un mentor se transforme en un proceso de coconstrucción, centrado en la mejora de prácticas educativas que se realizan en contextos educativos particulares. Desde esta mirada, se entenderá que las estrategias de asesoramiento del mentor harán alusión tanto a contenidos disciplinares y pedagógicos, como al apoyo en la instalación de las fases del proceso de mentoría, las tareas que deben desarrollarse en cada fase y los focos que emergen en cada una de las intervenciones.

En este punto es necesario definir el modelo educativo que sostiene el proceso de mentoría, ya que dicha acción permite establecer los lineamientos que definen cómo se observa y analiza la realidad. Vonk (1995), por su parte, al referirse a los elementos que debiera considerar un programa de formación de principiantes señala que, para el desarrollo de las competencias profesionales, es importante considerar el tiempo y el análisis del impacto de la formación en la inserción profesional. Asimismo, un programa debiera considerar tres dimensiones: la personal, la contextual y los conocimientos y destrezas del docente principiante. Por otro lado, Negrillo e Iranzo (2009) proponen que los contenidos de un programa de formación para profesores principiantes deben adaptarse a las necesidades reales manifestadas por los docentes noveles. En este sentido, un programa no debiese contar con contenidos estructurados de forma preestablecida ni homogeneizante, sino más bien con flexibilidad suficiente para reaccionar a situaciones problemáticas que emerjan en contextos educativos reales.

En el ámbito de las estrategias metodológicas, se sugiere realizar actividades guiadas de reflexión sobre la propia práctica, recogiendo la 
descripción de escenarios y contextos que incluya las argumentaciones sobre razones y sentimientos asociados a determinada actuación. Será preciso establecer dinámicas reflexivas y colaborativas, además de momentos de atención individual, que permitan fomentar un clima de intercambio y acogida en pos de potenciar valores y habilidades de cooperación como estrategia de apoyo.

Por otra parte, en su modelo de asesoramiento ${ }^{2}$, Lago y Onrubia (2011b, 2011a) plantean algunas tareas relacionadas con la importancia de considerar e integrar recursos discursivos por parte del mentor o asesor. Dichas tareas se vincularían con las formas de intervención durante el desarrollo de un proceso de asesoramiento (ver tabla 1).

Tabla 1.

Recursos discursivos del asesor planteados por Lago y Onrubia (2011a)

Presentar un problema o un subproblema nuevo.

Señalar una tarea a realizar.

Preguntar, pedir opinión, solicitar información o aclaración.

Aportar información complementaria respondiendo a una pregunta, complementando una intervención anterior y/o aclarando una duda.

Aceptar, repetir parte de la intervención para confirmar una interpretación, preguntar o comentar.

Relacionar, justificar y argumentar, incorporando reflexiones sobre la actividad de enseñanza y aprendizaje.

Conceptualizar, reelaborar y argumentar con un concepto, una aportación de otros o una justificación de una propuesta.

Formular una síntesis o proponer una conclusión.

Según estos autores, para una comunicación efectiva durante las actividades de asesoramiento es necesario que, en las intervenciones del asesor, se planifiquen y combinen distintos tipos de recursos discursivos que se ajusten a las características de las sesiones y a los

9 Denominado por sus autores como "modelo educacional constructivista de asesoramiento psicopedagógico", tiene por finalidad que los asesores (acompañantes) proporcionen herramientas y espacios a quienes asesoran (docentes y escuelas). Dicho modelo recoge diversas fuentes teóricas que pueden profundizarse en los escritos de Lago y Onrubia (2011a). Para fines de este estudio, el asesoramiento será considerado como una forma de acompañamiento. 
contenidos o ámbitos de mejora. Asimismo, proponen desarrollar variaciones de las secuencias de recursos a partir de preguntas y demandas de opinión, de aportación complementaria y de aceptación o confirmación (Lago y Onrubia, 2008). Conocer los recursos discursivos que puedan resultar más relevantes en cada fase puede ofrecer importantes recomendaciones a los asesores para mejorar su actuación en los procesos de asesoramiento (Roca, Armengol, Huerta y Onrubia, 2019).

Durante las sesiones de mentoría, también es posible identificar una serie de recursos comunicativos que utilizan profesores mentores y principiantes como fuente de argumentación y validación del conocimiento que se ha construido, o se construye, en los diferentes espacios educativos en los que se insertan. Estos recursos han sido denominados "invocaciones", y corresponden a enunciados que se sostienen o apoyan en diferentes elementos del conocimiento, lo que permite al hablante relacionar aquello que está hablando con elementos que justifican y validan su versión del conocimiento (Cubero et al., 2008). Según esta autora, el análisis de las invocaciones permite comprender cómo se interpretan, en los contextos de aula o de mentoría, los hechos o las explicaciones de las teorías científicas o disciplinares, la experiencia personal, etc. Existen cuatro tipos de invocación a: i) la autoridad de especialistas, conocimiento académico o profesional; ii) la experiencia y el conocimiento experiencial; iii) la verdad y, finalmente, iv) una ideología o un sistema de valores (Ibíd.). Dichas invocaciones podrían ubicarse en un continuo entre los recursos discursivos que tienden a producir un mayor o menor efecto distanciador entre los participantes. Por ejemplo, las invocaciones en que se incorpora el conocimiento académico tienden a producir distanciamiento, a diferencia de las invocaciones relacionadas con el conocimiento experiencial que tienen un efecto aproximador.

\section{Metodología}

El enfoque metodológico utilizado en este estudio es cualitativo, de carácter exploratorio y descriptivo-interpretativo. El diseño se ha centrado en el estudio de cuatro casos, compuestos cada uno por un mentor y un docente principiante, para realizar una indagación 
detallada y profunda del proceso de mentoría (Yin, 1989). Como fue mencionado, a partir de esta investigación se pretende describir las reflexiones que promueven, los saberes que movilizan y las estrategias que utilizan los mentores para facilitar el proceso de mentoría que se encuentra en una etapa inicial.

Para llevar a cabo el estudio se analizaron las siguientes fuentes de información:

a. Datos de situaciones de reflexión conjunta (sesiones de mentoría) entre mentores y sus docentes principiantes. Durante el proceso de acompañamiento, el mentor sostiene reuniones con el docente principiante, con el fin de apoyar y seguir su proceso formativo y reflexivo. Para este estudio se analizaron los audios de la primera sesión de mentoría.

b. Información producida en la entrevista en profundidad, realizada a mentores y a docentes principiantes al inicio del acompañamiento. Esta entrevista se realiza para profundizar en los fundamentos y marcos interpretativos que subyacen en los procesos de acompañamiento que realizan los mentores, los saberes que se relevan, los aspectos contextuales y relacionales que reconocen, y las acciones que declaran realizar durante las mentorías.

Para el procesamiento de la información se procedió a transcribir las sesiones y entrevistas y, posteriormente, se realizó un montaje en el software de análisis cualitativo Atlas.ti. 8.4. La técnica utilizada para el análisis de las entrevistas fue el análisis temático (Braun \& Clarke, 2006; Willig, 2013), que permite reconocer y ordenar información en cuanto a su contenido y significado, e identificar los temas más relevantes que construyen y dan sentido al fenómeno estudiado. Para definir los temas se utilizó un enfoque inductivo-deductivo y se establecieron categorías de análisis. Luego se cruzaron y relacionaron las categorías con el marco teórico desarrollado. Para definir los temas sobre los cuales versaban las sesiones de mentoría se procedió a identificar episodios, previa confección de un protocolo de análisis. En el caso de las estrategias utilizadas por el mentor para acompañar al docente principiante, se tomó como referencia el modelo de asesoramiento planteado por Lago y Onrubia (2011a, 2011b). Finalmente, para dar cuenta de 
los marcos interpretativos y saberes que mentores y principiantes relevan y movilizan durante las reuniones de mentoría, se procedió a realizar un nuevo análisis de los datos, utilizando los dispositivos discursivos de invocación establecidos por Cubero et al. (2008). Para ello se utilizó un protocolo que incluía los tipos de recursos discursivos e invocaciones definidas por dichos autores. Con el fin de resguardar la confiablidad, se utilizaron algunas estrategias cualitativas, la principal referida al proceso de triangulación en la identificación de los episodios, las temáticas de las sesiones y los recursos discursivos (LeCompte \& Preissle, 1982; Miguélez, 2006). Para realizar esta triangulación, dos investigadores examinaron los datos y, en función de los protocolos, identificaron las categorías y temáticas emergentes. De esta forma se buscó que, en los casos en los que no hubiese consenso, pudiera aportar un tercer investigador para definir la categoría en cuestión.

\subsection{Caracterización de la muestra}

La muestra comprende cuatro mentores con su correspondiente docente principiante. Los criterios para la selección de los mentores consideraron que se encontraran adscritos al registro público del Cpeip, residieran en la Región Metropolitana, estuvieran realizando mentorías en el sistema escolar y pertenecieran a comunas distintas.

Los casos que quisieron participar voluntariamente en este estudio pertenecen a distintas comunas de la Región Metropolitana y desempeñan las mentorías en establecimientos de dependencia municipal. Algunas características adicionales son:

- Pertenecen a las comunas de Quinta Normal, Santiago Centro, Renca y Vitacura.

- Dos mentores son mujeres y dos son hombres.

- Dos docentes mentores y principiantes trabajan en el mismo establecimiento de la misma comuna, y dos trabajan en distintos establecimientos de la misma comuna.

- Un solo caso cuenta con un año de antigüedad como mentora. En el resto de los casos los mentores se desempeñan por primera vez en este rol. 
- Todos los docentes mentores son profesores de educación general básica y acompañan a docentes principiantes mujeres.

- Todos los docentes mentores han aprobado su proceso de formación en mentoría y fueron formados en la misma institución formadora ${ }^{10}$.

Tabla 2 .

Sintesis de caracterización de los casos

\begin{tabular}{|c|c|c|c|c|}
\hline Caso & Participante & Formación & $\begin{array}{c}\text { Años experiencia } \\
\text { laboral en } \\
\text { educación }\end{array}$ & $\begin{array}{l}\text { Experiencia } \\
\text { en mentoría }\end{array}$ \\
\hline $\begin{array}{l}\text { Caso } 1 \\
\text { Santiago }\end{array}$ & Mentor & $\begin{array}{l}\text { Profesor educación } \\
\text { general básica, } \\
\text { especialidad }\end{array}$ & 8 & Primer año \\
\hline \multirow{2}{*}{$\begin{array}{l}\text { Docentes } \\
\text { trabajan en } \\
\text { el mismo } \\
\text { establecimiento }\end{array}$} & & Historia & & \\
\hline & Principiante & Profesora de Inglés & Ninguna & Ninguna \\
\hline $\begin{array}{l}\text { Caso } 2 \\
\text { Quinta Normal }\end{array}$ & Mentor & $\begin{array}{l}\text { Profesora educación } \\
\text { general básica }\end{array}$ & 11 & Primer año \\
\hline $\begin{array}{l}\text { Docentes } \\
\text { trabajan en } \\
\text { distintos } \\
\text { establecimientos }\end{array}$ & Principiante & $\begin{array}{l}\text { Profesora de } \\
\text { educación } \\
\text { diferencial }\end{array}$ & $\begin{array}{l}\text { Remplazo en } \\
\text { establecimientos } \\
\text { educacionales }\end{array}$ & Ninguna \\
\hline $\begin{array}{l}\text { Caso } 3 \\
\text { Renca }\end{array}$ & Mentor & $\begin{array}{l}\text { Profesor educación } \\
\text { general básica, } \\
\text { especialidad }\end{array}$ & 30 & Primer año \\
\hline \multirow{2}{*}{$\begin{array}{l}\text { Docentes } \\
\text { trabajan en } \\
\text { el mismo } \\
\text { establecimiento }\end{array}$} & & Matemáticas & & \\
\hline & Principiante & Profesora de Inglés. & Ninguna & Ninguna \\
\hline $\begin{array}{l}\text { Caso } 4 \\
\text { Vitacura }\end{array}$ & Mentor & $\begin{array}{l}\text { Profesora educación } \\
\text { general básica. } \\
\text { Magíster en }\end{array}$ & 12 & Segundo año \\
\hline \multirow{2}{*}{$\begin{array}{l}\text { Docentes } \\
\text { trabajan en } \\
\text { distintos } \\
\text { establecimientos }\end{array}$} & & $\begin{array}{l}\text { Matemáticas. } \\
\text { Formadora de } \\
\text { mentores }\end{array}$ & & \\
\hline & Principiante & $\begin{array}{l}\text { Profesora educación } \\
\text { general básica }\end{array}$ & Ninguna & Ninguna \\
\hline
\end{tabular}

10 La mayoría de los mentores adscritos al registro público del Cpeip, que pertenecen a la Región Metropolitana, han sido formados por la Universidad San Sebastián. El enfoque formativo de esta institución se caracteriza por centrarse en el sujeto que se forma; de esta manera se espera que, a partir del propio conocimiento, pueda contribuir de mejor forma a la construcción profesional de los profesores principiantes (Fuentealba y Jara-Chandía, 2017). 
Para resguardar los aspectos éticos de la investigación, el proyecto fue revisado y aprobado por el Comité de Ética de la Universidad Alberto Hurtado. Asimismo, los participantes firmaron un documento de consentimiento informado.

\section{Primeros hallazgos}

Los hallazgos se obtienen en un contexto de fuerte movilización social, condición que influyó en la sistematicidad de los encuentros del mentor con el principiante. Es relevante precisar que estos hallazgos no pretenden ser concluyentes, sino más bien responden a una primera aproximación que busca caracterizar el proceso de mentoría en su fase inicial.

Si bien los objetivos del estudio apuntaban directamente a la indagación sobre el proceso de mentoría, profundizar sobre el contexto en que se sitúan dichos programas emergió como un elemento fundamental en el discurso de los actores. Otro elemento destacado se vinculó a los aspectos relacionales que median la relación mentor-principiante. Asimismo, el estudio ahondó en las concepciones sobre mentorías y cómo estas se relacionan con las acciones de acompañamiento implementadas. En este sentido, se presentan dos grandes apartados referidos arelacionados con a) concepciones del mentor y del principiante sobre elementos de la mentoría y su contexto, y b) organización y desarrollo del proceso de mentoría. En la primera sección se ahondará, entre otros temas, en la concepción de las mentorías, las dificultades y expectativas asociadas al proceso, y en las condiciones que favorecerían su implementación. En la segunda se dará cuenta de la organización real efectuada durante las sesiones, las conversaciones y reflexiones suscitadas, y los marcos interpretativos, saberes y recursos discursivos que los mentores movilizan en dichas sesiones.

\subsection{Concepciones del mentor y del principiante sobre elementos de la mentoría y su contexto}

A partir de las entrevistas, emergen diversos aspectos contextuales y relacionales que permiten situar el trabajo de las duplas en sus 
contextos educativos particulares. A continuación, se mencionan los elementos más destacados por los actores:

\section{Concepción y rol de las mentorías}

La mentoría emerge como una instancia favorecedora del desarrollo de habilidades profesionales, constituyéndose en un espacio de aprendizaje entre pares que brinda apoyo a quienes se insertan en los establecimientos. En este sentido, se instala no solo como un apoyo al proceso de inserción al aula, sino al sistema educativo en su conjunto, por lo que las mentorías se consideran como un proceso necesariamente anclado al contexto escolar en el que emergen. No son una "receta" al principiante, sino más bien un proceso constitutivo de los contextos en que se lleva a cabo. Se destaca la importancia de concebir la mentoría como un proceso de crecimiento para ambos docentes.

Para mí la mentoría ha sido un proceso de aprendizaje. Yo creo que lo que más tengo de mentor es como la motivación a aprender en conjunto, porque, en definitiva (...), no hay una receta del mentor, sino más bien de un acompañante, del compartir experiencias, el de ir creciendo juntos, también. (Entrevista inicial docente mentor. Caso 1)

Las competencias y disposiciones que se busca fomentar en los principiantes, según ambos docentes, se organizan en dos ámbitos. En primer lugar, se menciona el desarrollo de saberes pedagógicos ${ }^{11}$ que se movilizan en los distintos espacios del aula, como son las habilidades socioemocionales, que permiten a los principiantes lidiar con la incertidumbre de ingresar a un espacio laboral desconocido y desafiante, y, a su vez, resolver conflictos administrativos y relacionales que emergen con los actores de la comunidad escolar. En segundo término, el conocimiento didáctico y curricular que permite resolver situaciones problemáticas en el aula, favorecer el

11 Acuñaremos la definición de "saberes pedagógicos" entregada por la Comisión Nacional sobre la Formación Inicial Docente (2005), que los considera como todos aquellos saberes relacionados con cómo se produce el aprendizaje y los factores que intervendrían para su desarrollo en un contexto general. En esta definición se considera a los estudiantes, a la escuela y al curriculum. También se vinculan con la interacción en el aula, el conocimiento, la comprensión del contenido de la especialidad y su didáctica. 
30 PRÁCTICAS DE MENTORÍA PARA LA INDUCCIÓN DE DOCENTES PRINCIPIANTES: ANÁLISIS DE CUATRO CASOS CHILENOS AL INICIO DEL PROCESO - S. Gorichon, M. Salas, M. Araos, M. Yáñez, A. Rojas-Murphy, G. Jara-Chandía

conocimiento de la cultura y del sistema escolar, y proveer soluciones pertinentes al contexto en que se insertan.

\section{Las dificultades que enfrentan los docentes principiantes}

Mentores y principiantes plantean que una de las dificultades que enfrentan los noveles se relacionan con sus expectativas acerca de las acciones que efectivamente pueden realizar en la escuela y en sus aulas. La institución educativa es percibida como un espacio complejo, en el que se sienten inseguros de las respuestas que pueden ofrecer, considerando la cultura de los contextos socioculturales en que se insertan. Algunos mentores también consideran que existe un sentimiento de frustración por parte del principiante, al observar la distancia entre los conocimientos aprendidos en la universidad y los requerimientos del sistema escolar.

Yo creo que, por una parte, el tema de la frustración del docente principiante [se refiere a su expectativa] que es muy alta, frente a las primeras aproximaciones, por decirlo de alguna manera, o primeras experiencias laborales. Creo que determina mucho cómo tú eres capaz de afrontar tu primer año laboral, el resultado de lo que vas a tener más adelante, porque (...) el tema de que los estudiantes de pregrado o los profesores iniciales vienen tan idealizados de un sistema que no existe, al cual tú debes tratar como de modificar o como de moldear a la realidad donde te vas a ver envuelto. Todo eso ideológico que te plantearon, llevarlo a lo concreto es súper complejo. (Entrevista inicial mentor. Caso 1)

Pucha, a mi parecer son varias las dificultades iniciales. Desde el contraste con los aprendizajes que uno viene desde la universidad y como la puesta en práctica, eso es muy fuerte. (Entrevista inicial docente principiante. Caso 3)

\section{Expectativas y desafíos que manifiestan del proceso de mentorías: las agendas}

Una de las principales expectativas de los mentores, en su trabajo con los principiantes, es evitar su deserción y que puedan reconocer la importancia social de su labor dentro de la comunidad educativa. 
Entonces, la primera es que ella no abandone, que descubra lo bonito que es la profesión, con todos sus bienes y males que tiene (...). Que al final de la meta están los chiquillos que ves de nuevo, siendo ingenieros (...), doctores, abogados, tenemos de todo. Entonces esa satisfacción, lograr que también la sepa. (Entrevista inicial mentor. Caso 2)

Asimismo, los mentores esperan contribuir al desarrollo profesional de los principiantes a través de la generación de espacios de aprendizaje colaborativos, en los que ambos puedan crecer y compartir sus experiencias y conocimientos. Por su parte, los principiantes manifiestan querer aprender de la experiencia de sus mentores, valorando estrategias que les permitan generar un clima propicio para el aprendizaje, desarrollar estrategias pedagógicas contextualizadas, elaborar un diseño de clases atingente o, bien, resolver situaciones complejas en el aula.

Me gustaría aprender mucho de mi profesor, porque sé que tiene como, como harta experiencia y también harto respeto, como que los chicos lo respetan harto, así como que siento que quizás a mí tampoco (...), no es que diga que a mí me falten el respeto, pero como que hay algo más ¿cachai? Son los años también. (Entrevista inicial docente principiante. Caso 1)

Finalmente, esperan que el espacio de las mentorías constituya un lugar en el que se sientan seguros, permitiéndoles generar una mayor autoconfianza para enfrentar las situaciones propias de su inserción profesional.

Competencias y saberes necesarios para acompañar a los docentes principiantes

Las competencias que debería poseer el mentor se relacionan con aspectos socioafectivos y comunicativos. Asimismo, se valora que posean conocimientos pedagógicos, disciplinares, didácticos y del sistema educativo en general, y capacidad para hacer progresar al principiante mediante la reflexión. En cuanto a los aspectos socioemocionales que los principiantes relevan de los mentores, se encuentra la disposición a acompañarlos y de ayudarlos a enfrentar los requerimientos emergentes. 
32 PRÁCTICAS DE MENTORÍA PARA LA INDUCCIÓN DE DOCENTES PRINCIPIANTES: ANÁLISIS DE CUATRO CASOS CHILENOS AL INICIO DEL PROCESO - S. Gorichon, M. Salas, M. Araos, M. Yáñez, A. Rojas-Murphy, G. Jara-Chandía

Yo creo que debe tener una disposición al cien, y tiempo para poder resolver todas mis dudas, para acompañarme. Creo que una persona que no tenga disposición no debería ser mentor. La paciencia igual, que eso es súper importante. (Entrevista inicial docente principiante. Caso 2)

En relación con aspectos pedagógicos, los mentores enfatizan en que el acompañamiento debiese focalizarse en desarrollar procesos reflexivos sobre las prácticas del principiante, permitiéndole reconocer sus aciertos y dificultades. Esto repercutirá posteriormente en su relación con la comunidad educativa y en los aprendizajes que adquirirán sus estudiantes.

Yo creo que un buen mentor es el que lleva a la otra persona a que reflexione sobre sus prácticas pedagógicas (...). Que se dé cuente que lo está haciendo bien, que aprenda a conocer sus debilidades y fortalezas ¿cierto? (...) Adaptarse con sus directivos, con sus colegas, y esto va a ir bien encaminado y va a resultar excelente con los niños y para ella como persona también. (Entrevista inicial mentora. Caso 4)

Condiciones que favorecen u obstaculizan el desarrollo de las mentorías

Según los docentes, uno de los aspectos que incidiría en el desarrollo de las mentorías es que las duplas pertenezcan al mismo contexto educativo, ya que esto permitiría realizar un acompañamiento situado y facilitaría la inserción del principiante, acercándose a las lógicas de funcionamiento interno del establecimiento, del clima organizacional y del contexto escolar en general. Otro aspecto que influiría sería la disposición de los equipos directivos y de las comunidades escolares para acoger y apoyar el proceso de inducción. Se identifica como elementos favorecedores la disponibilidad de espacios y tiempos para reunirse y la valoración de la labor contextualizada del mentor.

Entonces, yo también creo que debería ser considerado, no sé, al menos dos horas dentro de la hora de trabajo para ver todo el proceso de sala, para el acompañamiento de sala, para ver los errores o ver cómo lo estoy haciendo, porque uno igual se enfrenta al curso, principiante, 
al contexto nuevo; son diferentes contextos, igual a nivel comunal. (Entrevista inicial docente principiante. Caso 2)

También se plantea como obstaculizador la incerteza contractual del principiante durante su primer año, y la escasa claridad de su situación laboral para el año siguiente, lo que afectaría su estado emocional y la continuidad del proceso de mentoría.

Igual tiene esa incertidumbre que no sabe qué va a pasar para el próximo año, porque en el sentido de la condición es complejo, porque ella está reemplazando a una profe que está con licencia. Entonces eso la angustia a ella. (Entrevista inicial mentor. Caso 1)

El conocimiento del sistema escolar que posee el mentor resulta fundamental para una mejor inserción de los principiantes en los establecimientos educacionales; especialmente del sistema municipal y de las políticas públicas que orientan el quehacer de las escuelas.

Pasa por el saber cómo hablar con los pares, cómo hablar con los jefes, cómo desenvolverse bien en una reunión con padres y apoderados, cómo salir adelante en los momentos difíciles, que puedes tener un mal rato con tus jefes, con tus amigos, con la mesa, porque tener mesa, puesto, es importante, y convivir ahi y al otro día volver a tener ese lugar en la mesa. (Entrevista inicial docente principiante. Caso 2)

\subsection{Organización y desarrollo de las mentorías}

\section{Organización general del proceso}

El proceso de mentoría se organiza contemplando la propuesta diseñada por el Cpeip, que entrega orientaciones y objetivos, establece tiempos para la realización de las actividades propuestas y también incluye los acuerdos del mentor y principiante, que deciden conjuntamente cómo realizarán el acompañamiento. La propuesta organizativa del Cpeip considera una planificación anual y una bitácora, en la que se registran las sesiones, que contempla seis horas semanales de acompañamiento al principiante. 
Se señalan tres fuentes de información que permiten identificar y ajustar los apoyos provistos por la mentoría: la percepción del principiante, las observaciones de aula realizadas por el mentor y, en menor medida, las sugerencias del director o jefe de la Unidad Técnico Pedagógica del establecimiento. Los cuatro casos informan que en la primera sesión se aplica una entrevista o se formulan preguntas con el fin de indagar las necesidades y apoyos del principiante. La planificación de visitas al aula, en los cuatro casos, se realiza de forma acordada y en el colegio del principiante. Los mentores señalan que, en su retroalimentación, entregan distintos recursos para apoyar el proceso de implementación de los principiantes, tales como uso y elaboración de material didáctico, y orientaciones e ideas para realizar las clases previstas. En el caso de los docentes que comparten colegio se facilita el apoyo oportuno, debido a la cercanía que se produce entre mentor y principiante, lo que permite contener a los aprendices y reflexionar sobre las situaciones emergentes de la práctica.

Finalmente, los mentores destacan la importancia de incorporar la dimensión socioafectiva al inicio de la sesión; interesarse por el estado personal, familiar y emocional del principiante resulta decisivo para el proceso de acompañamiento.

Bueno, al principio, primero que me contara cómo estaba, todos los días en realidad llego así: "¿Cómo ha estado tu semana? ¿Algún problema? ¿Tu familia? ¿Tu pololo?”, porque siempre me cuenta de su vida y de ahí ya partimos, como más menos si había tenido algún problema, no sé, algo que fuera importante para ella, todo lo que había pasado en la escuela. (Entrevista inicial mentora. Caso 4)

\section{Conversaciones y reflexiones en las mentorías}

Los mentores declaran que los aspectos sobre los cuales dialogan y reflexionan en las mentorías se relacionan fundamentalmente con aspectos personales del principiante, elementos administrativos y estrategias pedagógicas y didácticas, ajustadas a los contextos educativos particulares y a la cultura del establecimiento. La planificación de clases constituye una de las conversaciones más generalizadas de las sesiones de mentoría, abriendo el diálogo acerca 
de la preparación de la enseñanza en relación con las necesidades de los estudiantes. Si bien lo administrativo cobra un papel preponderante y recurrente de consulta, los mentores manifiestan que este apoyo constituye un elemento más bien técnico.

Porque era lo que conversamos la otra vez, el hecho de no saber llenar un libro, no saber colocar una anotación, cómo derivan un caso de un niño, son cosas que la universidad no te prepara, y que uno las va aprendiendo sobre la marcha, consultando, muchas veces comprometido también. (Entrevista inicial mentor. Caso 1)

Por su parte, los principiantes valoran que en las sesiones se trabaje en la identidad docente, lo que les permite tener mayor conciencia de las representaciones y creencias respecto de su quehacer profesional. Finalmente, se manifiesta que la reflexión constituye una capacidad clave que les permite aprender y mejorar sus prácticas docentes.

El aprendizaje que yo quiero entregar es la reflexión. Que ella reflexione, aprenda a reflexionar, que mire su práctica, que aprenda a descubrir que cada día uno puede mejorar. Que aprenda a preparar, a preparar sus clases. (Entrevista inicial mentor. Caso 2)

Al realizar un análisis de las sesiones iniciales de mentoría ${ }^{12}$ se identifican las siguientes temáticas que, en su mayoría, se vinculan con los elementos destacados en sus entrevistas:

- Estado socioemocional del principiante, con relación a los ámbitos personal y familiar, aula, contexto laboral y relaciones con sus pares.

- Definición del propósito de las mentorías y del rol del mentor.

- Definición de acciones que podrían realizarse en las mentorías: observación de otras prácticas de profesores y observación de clases del mentor.

12 Las sesiones iniciales de mentoría fueron grabadas por las mismas duplas y analizadas posteriormente a partir de un protocolo que permitía la definición de episodios, recursos discursivos e invocaciones. 
- Análisis de las prácticas, conocimiento docente y sugerencias de mejora con relación a:

- Diseño de planificaciones para el aula.

- Implementación, interacciones y normas de convivencia en aula.

- Evaluación: acciones de monitoreo para verificar avances y aprendizajes de los estudiantes.

- Marcos regulatorios e instrumentos de gestión escolar, tales como el Marco para la Buena Enseñanza, e instrumentos de gestión que se utilizan en el establecimiento educacional (PEI y PME) y su importancia como orientadores del quehacer docente.

- Valoración del proceso llevado a cabo al interior de las sesiones de mentoría y aprendizajes logrados por ambos docentes, vinculándolos al desarrollo identitario y al sello personal del principiante.

- Tareas administrativas para desarrollar en el establecimiento y/o dudas del principiante.

Las temáticas más abordadas en las sesiones por las duplas de mentoría fueron la indagación del estado socioemocional en que se encuentra el principiante, el análisis de sus prácticas y, finalmente, la valoración de la mentoría y los aprendizajes obtenidos. Se identificó en el caso 4 una amplia variedad de temáticas, especialmente relacionadas con la valoración del desempeño del principiante a partir de la descripción y reflexión de situaciones de la práctica.

Marcos interpretativos, saberes y recursos discursivos que los mentores relevan y movilizan en las sesiones

Otro aspecto que emergió de las sesiones de mentoría se relaciona con los marcos interpretativos y saberes que los mentores y principiantes movilizan durante las sesiones para justificar y legitimar sus ideas, afirmaciones y acciones. Se utilizó la matriz de dispositivos discursivos de invocación, establecidos por Cubero et. al., (2008), a través de la cual el hablante puede invocar diferentes elementos del conocimiento académico y experiencial, de la verdad o de su propio sistema de valores e ideología para apoyar sus enunciados. 
Tabla 3.

Frecuencia de invocaciones de los casos

\begin{tabular}{|c|c|c|c|c|c|c|}
\hline \multirow{2}{*}{$\begin{array}{l}\text { Caso/temática } \\
\text { sesión }\end{array}$} & \multirow[t]{2}{*}{ Participante } & \multicolumn{4}{|c|}{ Frecuencia invocaciones } & \multirow{2}{*}{$\begin{array}{c}\text { Total } \\
\%\end{array}$} \\
\hline & & $\begin{array}{l}\text { Conocimiento } \\
\text { académico }\end{array}$ & $\begin{array}{l}\text { Conocimiento } \\
\text { experiencial }\end{array}$ & $\begin{array}{c}\text { A la } \\
\text { verdad }\end{array}$ & $\begin{array}{l}\text { Ideología } \\
\text { o sistema } \\
\text { de valores }\end{array}$ & \\
\hline \multirow{2}{*}{$\begin{array}{l}\text { Caso l } \\
\text { Retroalimentación } \\
\text { de una clase } \\
\text { observada y ajuste } \\
\text { de mejora }\end{array}$} & Mentor & $12,5 \%$ & $0,0 \%$ & $0,0 \%$ & $0,0 \%$ & $12,5 \%$ \\
\hline & Principiante & $37,5 \%$ & $37,5 \%$ & $6,3 \%$ & $6,3 \%$ & $87,5 \%$ \\
\hline \multirow[b]{2}{*}{$\begin{array}{l}\text { Caso } 2 \\
\text { Marcos } \\
\text { regulatorios del } \\
\text { establecimiento } \\
\text { (PME y PEI), } \\
\text { cultura escolar y } \\
\text { ajustes al plan de } \\
\text { inducción }\end{array}$} & Mentor & $46,7 \%$ & $31,7 \%$ & $20,0 \%$ & $0,0 \%$ & $98,3 \%$ \\
\hline & Principiante & $0,0 \%$ & $1,7 \%$ & $0,0 \%$ & $0,0 \%$ & $1,7 \%$ \\
\hline \multirow{2}{*}{$\begin{array}{l}\text { Caso } 3 \\
\text { Valoración de la } \\
\text { clase observada } \\
\text { y sentimientos } \\
\text { asociados }\end{array}$} & Mentor & $35,7 \%$ & $7,1 \%$ & $0,0 \%$ & $0,0 \%$ & $42,9 \%$ \\
\hline & Principiante & $28,6 \%$ & $14,3 \%$ & $14,3 \%$ & $0,0 \%$ & $57,1 \%$ \\
\hline \multirow[b]{2}{*}{$\begin{array}{l}\text { Caso } 4 \\
\text { Análisis de una } \\
\text { planificación, } \\
\text { interacción } \\
\text { entre pares y } \\
\text { construcción de su } \\
\text { rol como profesor }\end{array}$} & Mentor & $21,5 \%$ & $5,7 \%$ & $6,7 \%$ & $0,5 \%$ & $34,4 \%$ \\
\hline & Principiante & $13,9 \%$ & $25,4 \%$ & $13,9 \%$ & $12,4 \%$ & $65,6 \%$ \\
\hline
\end{tabular}

En general, se observa que, en las sesiones, los principiantes utilizan más las invocaciones como elementos discursivos para dar fundamentación a sus afirmaciones, descripciones y acciones (53,5\%); mientras que los mentores alcanzan una frecuencia menor (46,5\%). Los mentores emplean preferentemente el conocimiento académico para argumentar o fundamentar sus afirmaciones o acciones en el proceso de mentoría; con baja frecuencia se basan en su conocimiento experiencial.

En el caso de los principiantes, en los casos 1, 3 y 4 (en que conversan acerca del análisis y valoración de la práctica docente a partir de observación de clases, análisis de planificación e interacción con pares) se evidencia mayor frecuencia de invocaciones, como también mayor diversidad de tipos de invocaciones, acudiendo 
principalmente a las académicas y experienciales. En el caso 2, que versa sobre un diálogo acerca de marcos regulatorios del establecimiento, solo se invoca al conocimiento experiencial. Cabe señalar que, en el caso 4, cuya mentora presenta mayor antigüedad en mentoría, ambas docentes legitiman sus discursos y acciones invocando, además del conocimiento académico y experiencial, a la verdad e ideología, o al sistema de valores. Solo en los casos 1 y 4 las principiantes invocan a la ideología o sistema de valores.

También se observa que, mayoritariamente, es el mentor quien conduce las conversaciones y temáticas de las sesiones de mentoría, observándose en general el uso de los siguientes recursos discursivos $^{13}$ :

- Pregunta, pide opinión y/o solicita información para clarificar el contexto, la actividad o temática.

- Pregunta, pide opinión y/o solicita información para profundizar en la situación o temática.

- Aporta información, ya sea complementando una intervención anterior o aclarando alguna duda planteada por el principiante.

- Parafrasea las aportaciones, comentarios o ideas planteadas por el principiante.

- Argumenta incorporando nuevos elementos al análisis de las situaciones.

- Reacciona a los aportes del principiante mediante reelaboraciones o reconceptualizaciones.

- Atribuye una etiqueta o un concepto a aspectos que se describen del contexto, situación o temática.

En el caso 4, que se refiere a quien posee más antigüedad como mentora, se manifiesta la utilización de todos los recursos discursivos; en mayor cantidad los relacionados con atribución de conceptos, conceptualización, reelaboraciones y solicitud de preguntas de profundización. En el caso 1, que corresponde a una sesión de retroalimentación de observación de clases, se constata mayor uso de

13 Los recursos discursivos serán entendidos como un conjunto de recursos semióticos y estrategias discursivas que utiliza el mentor en las intervenciones que realiza para favorecer el desarrollo de los propósitos de las mentorías en sus distintas fases (Lago y Onrubia, 2011). 
recursos discursivos relacionados con argumentación, incorporando nuevos elementos al análisis de situaciones. Finalmente, en los casos 2 y 3 existe mayor presencia de recursos discursivos relacionados con preguntar, pedir opinión y/o solicitar información para clarificar o profundizar.

\section{Discusiones y proyecciones}

Los resultados de los cuatro casos presentados están permeados por la naturaleza de la mentoría que se encuentra en su fase inicial; por ello, no pretenden ser concluyentes, sino más bien constituyen una primera aproximación comprensiva a las prácticas de mentoría en esta fase. Para caracterizar las prácticas del mentor fue necesario analizar y dar cuenta de los aspectos contextuales que emergieron reiterativamente en los discursos de mentores y principiantes.

Sobre la concepción que se tiene de las mentorías, los docentes coinciden en concebirla como un proceso de acompañamiento situado, que responde a las características de los principiantes y al contexto escolar en que se enmarcan. Los hallazgos de este estudio refrendan lo descrito por la literatura respecto de las dificultades que presentarían los principiantes en su inserción profesional, las que se vincularían con los desafíos experimentados frente a situaciones problemáticas que emergen de la práctica, o bien con la complejidad de interpretar y comprender la cultura escolar de los establecimientos educativos en que se insertan mentores y principiantes.

Las expectativas de los mentores y principiantes coinciden en la relevancia de formar buenos docentes que se destaquen como miembros activos de una comunidad educativa. Los principiantes declaran, en general, la necesidad de contar con mayores herramientas pedagógicas y didácticas que les permitan resolver situaciones complejas en el aula; mientras los mentores muestran preocupación por proveer espacios de análisis y reflexión sobre temáticas que pudiesen tensionar a los principiantes, haciendo necesario relevar el carácter formativo de las "preguntas reflexivas", específicamente para desarrollar la capacidad de tomar decisiones autónomas por parte del principiante en su ejercicio profesional. 
40 PRÁCTICAS DE MENTORÍA PARA LA INDUCCIÓN DE DOCENTES PRINCIPIANTES: ANÁLISIS DE CUATRO CASOS CHILENOS AL INICIO DEL PROCESO - S. Gorichon, M. Salas, M. Araos, M. Yáñez, A. Rojas-Murphy, G. Jara-Chandía

Por otra parte, este estudio nos permite identificar aspectos valorados por las duplas respecto de las competencias que debiera poseer un mentor para llevar a cabo este acompañamiento docente. Los principiantes relevan la visión de un mentor support (Malderez \& Wedell, 2007), caracterizada por su disposición a la escucha y por establecer un vínculo personal, facilitando la generación de espacios de confianza. Aquí se reconoce que un buen mentor debiese ser un docente con experiencia en el sistema escolar y con fuerte dominio pedagógico, didáctico y de la cultura escolar. Este conocimiento, junto con la práctica reflexiva, se presentan como aspectos indispensables para la progresión de los principiantes.

Los focos temáticos que se conversan y reflexionan en las sesiones de mentorías son variados y con propósitos distintos. Si bien la mayoría de estos coinciden con las dificultades planteadas por los principiantes y con los temas sugeridos por el Cpeip, en esta fase inicial emerge con mayor fuerza la indagación que realiza el mentor, al comienzo de cada sesión, acerca del estado emocional del principiante; estrategia altamente valorada por este último, ya que permite fundar espacios de confianza que incidan en el establecimiento de relaciones colaborativas.

Asimismo, es posible observar distintos tipos de recursos discursivos que utilizan los mentores en el diálogo con el principiante - Se observa una preponderancia de preguntas orientadas a clarificar información, en desmedro de otras que buscan profundizar en las temáticas abordadas, al igual que una fuerte tendencia a reaccionar a las aportaciones del principiante, incorporando comentarios o análisis sobre las situaciones de la práctica. En menor medida se aprecian recursos discursivos relacionados con parafrasear los comentarios u opiniones que surgen del aprendiz. Aquellos recursos discursivos de atribución de conceptos y reelaboraciones conceptuales, utilizados por el mentor a partir de lo que describe el principiante de su práctica, constituyen los recursos menos utilizados, a excepción de la mentora con más antigüedad que sí acude a estos recursos de manera frecuente.

Se constata que, mayoritariamente, los mentores utilizan invocaciones de carácter académico para fundamentar sus ideas 
y acciones; a diferencia de la mentora, con mayor antigüedad en mentoría, que realiza invocaciones al conocimiento experiencial. Al respecto, sería interesante contar con mayor evidencia de las distintas fases del proceso de mentoría, con el fin de comprobar si efectivamente determinados recursos discursivos contribuyen a potenciar procesos reflexivos que promuevan la mejora y el desarrollo de la autonomía del principiante (Lago y Onrubia, 2008; Roca, 2018), y si los tipos de invocaciones producen o no un efecto distanciador o aproximador entre el mentor y el principiante (Cubero et al., 2008).

A partir del análisis realizado, se observa cierta tensión entre el discurso declarativo del mentor, plasmado en las entrevistas, y las acciones que efectivamente desarrolla en los espacios de interacción con el profesor principiante. En este sentido, sería interesante seguir profundizando en la tensión entre el discurso relacionado con las creencias y percepciones sobre las mentorías, y las acciones que allí se emprenden. Uno de los primeros aspectos se relaciona con la forma de intervención que se adoptaría en los procesos de mentoría y su vínculo con un modelo de transmisión y reproducción de la información, o más bien con un enfoque de tipo constructivista (Ruffinelli, 2020). En las sesiones de mentoría es posible vislumbrar características de ambos modelos; sin embargo, dado que el proceso todavía se encuentra en una etapa inicial, no es posible constatar con claridad si dicha forma de acompañar constituirá una constante a lo largo de todo el proceso o bien variará en función de las fases de mentoría en que se encuentren mentor y docente principiante. Al respecto, los modelos de acompañamiento y asesoramiento que siguen una perspectiva constructivista plantean una serie de elementos que constituyen desafíos respecto de la forma de estructurar y organizar los procesos de mentoría, y que, a su vez, permiten avanzar en una definición que considere la resolución conjunta de los problemas y dificultades que se desea abordar entre docente mentor y principiante. Lo anterior demanda cautelar que no solo se aborden contenidos orientados hacia la mejora, sino también se defina cómo será el proceso de colaboración y corresponsabilidad que exigiría una forma de acompañamiento con un enfoque constructivista (Lago y Onrubia 2011a). 
Considerando los discursos de mentores y principiantes, es posible sostener que la mayoría de las sesiones de mentorías se organizan en función del plan y de las orientaciones sugeridas por el Cpeip. Sin embargo, el mentor que ha ejercido más tiempo este rol y que, además, cuenta con experiencia como formador de mentores, muestra una mayor flexibilidad respecto de esta propuesta, al generar nuevas estructuras que se relacionan con la necesidad de desarrollar un trabajo situado y pertinente. Esto permite sugerir que la diferencia que presenta la mentora con mayor antigüedad respecto de otros mentores se debe a esta experiencia metacognitiva sostenida en el tiempo.

A partir de lo mencionado, surgen una serie de interrogantes relacionadas con el proceso de formación de los mentores ¿Qué aprendizajes debieran incorporarse en la formación de los mentores a partir del reporte de las prácticas de mentorías? ¿Cuánto debe permanecer un mentor en formación para desarrollar y consolidarse en su rol? ¿Resulta suficiente la formación que reciben para desarrollar un proceso reflexivo que incida en el mejoramiento de su práctica? Lo anterior demanda un constante replanteamiento y ajuste del proceso formativo de mentores, como también de la posibilidad de considerar un acompañamiento a posteriori que les permita seguir desarrollando su labor.

Los hallazgos de este estudio permiten sugerir que la conducción de un proceso de acompañamiento se ve favorecido por las características personales del mentor, su experiencia respecto al proceso de inducción y de mentoría, y por las oportunidades de formación que posee en distintas esferas. En este punto sería interesante pensar la mentoría como una oportunidad de desarrollo profesional, tanto para el mentor como para el principiante, independiente del momento de la trayectoria profesional en que se encuentren. No hay que perder de vista que el mentor también es un docente que está empezando en este proceso de acompañar, siendo en ese escenario también un principiante. Sería interesante considerar un espacio resguardado por la política nacional o local que permitiera al mentor seguir desarrollando y madurando su acompañamiento a los principiantes. En relación con los programas 
de inserción profesional y laboral para principiantes, cabe preguntarse cuán prescriptivos debieran ser y qué grado de autonomía debiera tener este docente experimentado para tomar decisiones formativas en relación con el acompañamiento.

Desde los discursos de mentores y principiantes se evidencia que el proceso de mentoría está mediado por condiciones contextuales y de la cultura escolar que pueden favorecer u obstaculizar su desarrollo. Coinciden en lo vital que resulta ser acogido en un espacio escolar que promueva la colaboración y que éste sea liderado por equipos de gestión que valoren y legitimen los procesos de mentoría a través de la designación de tiempos y espacios exclusivos para su realización. Acompañar al docente principiante no solo debe depender de la labor del mentor, sino también de una comunidad educativa que lo acoja y apoye de manera activa. Lo anterior demanda mayor socialización de los procesos de inducción y mentoría en los contextos educativos, definición clara de los roles y funciones de los equipos directivos, como también de la promoción, al interior de los nuevos servicios locales, de orientaciones y acciones que favorezcan una cultura de colaboración y apoyo para los profesores que se inician en la docencia.

Finalmente, la inestabilidad laboral surge como un aspecto a considerar en el resultado de los acompañamientos, pues dicha situación provoca en los principiantes gran incertidumbre, angustia e inseguridad, lo que podría incidir en su desempeño en el establecimiento y en la posibilidad de dar continuidad al trabajo de las duplas mentor-principiante. Por ello se requiere, de manera urgente, que los municipios y empleadores dispongan de condiciones y apoyos apropiados para llevar a cabo la mentoría, pues un escenario adverso podría generar un desincentivo para participar de estos procesos de acompañamiento docente. 
44 PRÁCTICAS DE MENTORÍA PARA LA INDUCCIÓN DE DOCENTES PRINCIPIANTES: ANÁLISIS DE CUATRO CASOS CHILENOS AL INICIO DEL PROCESO - S. Gorichon, M. Salas, M. Araos, M. Yáñez, A. Rojas-Murphy, G. Jara-Chandía

\section{Referencias}

Achinstein, B. \& Athanases, S. Z. (2005). Focusing new teachers on diversity and equity: Toward a knowledge base for mentors. Teaching and Teacher Education, 21(7), 843-862.

Athanases, S. Z. \& Martin, K. J. (2006). Learning to advocate for educational equity in a teacher credential program. Teaching and Teacher Education, 22(6), 627-646.

Ávalos, B. (2009). La inserción profesional de los docentes. Revista de Currículum y Formación Del Profesorado, 13(1), 43-59.

Ávalos, B. \& Valenzuela, J. P. (2016). Education for all and attrition/retention of new teachers: A trajectory study in Chile. International Journal of Educational Development, 49, 279-290. DOI: https://doi.org/10.1016/j. ijedudev.2016.03.012

Billingsley, B., Bettini, E. \& Jones, N. D. (2019). Supporting Special Education Teacher Induction Through High-Leverage Practices. Remedial and Special Education, 40(6), 365-379. DOI: https://doi. org/10.1177/0741932518816826

Bransford, J., Derry, S., Berliner, D., Hammerness, K. \& Beckett, K. (2005). Theories of learning and their roles in teaching. In L. DarlingHammond \& J. Bransford (Eds.), Preparing teachers for a changing world (pp. 40-87). San Francisco: Jossey-Bass.

Braun, V. \& Clarke, V. (2006). Using thematic analysis in psychology. Qualitative Research in Psychology, 3(2), 77 101. DOI: https://doi.org/https://www.tandfonline.com/doi/ pdf/10.1191/1478088706qp063oa?needAccess=true

Centro de Perfeccionamiento, Experimentación e Investigaciones Pedagógicas, Cpeip. (n.d.). Sistema Nacional de Inducción para docentes principiantes. Recuperado de http://portales.mineduc.cl/usuarios/ cpeip/doc/201505181200150.CUADERNO_DEL_MENTOR_ INTRODUCCION.pdf

Cisternas, T. (2016). Dificultades experimentadas por profesores principiantes de Educación Básica en sus prácticas de enseñanza y su relación con la Formación Inicial. Santiago de Chile: CIDE/Universidad Alberto Hurtado.

Cochran-Smith, M. (2003). The multiple meanings of multicultural teacher education: A concep-tual framework. Teacher Education Quarterly, 30(2), 7-26.

Crutcher, P. A. \& Naseem, S. (2016). Cheerleading and cynicism of effective mentoring in current empirical research. Educational Review, 68(1), 40-55. DOI: https://doi.org/10.1080/00131911.2015.1058749 
Cubero, R., Cubero, M., Santamaría, A., De La Mata, M., Ignacio, M. y Prados, M. (2008). La educación a través de su discurso. Prácticas educativas y construcción discursiva del conocimiento en el aula. Revista de Educación, 343, 71-104.

Díaz, C. y Bastías, C. (2013). Los procesos de mentoría en la formación inicial docente. Revista Internacional de Investigación en Ciencias Sociales, 9(2), 301-315.

Fuentealba, A. R. y Jara-Chandía, G. (2017). Acta de Congreso INVEDUC. La experiencia de la formación en la universidad San Sebastián. DOI: https://doi.org/10.1017/CBO9781107415324.004

Gaete, A., Navarrete, M. C., Pino, F. y Mansilla, D. (2017). Abandono de la profesión docente en Chile: Motivos para irse del aula y condiciones para volver * Teacher attrition in Chile: Reasons for leaving the classroom and conditions for coming back. Estudios Pedagógicos XLIII, 1, 123-138.

Hirmas, C. y Cortés, I. (2015). Estado del arte, investigaciones sobre formación práctica en Chile. Tensiones y desafíos. Santiago de Chile: Organización de Estados Americanos para la Educación, la Ciencia y la Cultura.

Huberman, M., Gonauer, M. M. \& Marti, J. (1993). The Lives of Teachers. New York: Teachers College.

Iafrancesco, V. (2003). La investigación en educación y pedagogía: fundamentos y técnicas. Bogotá: Cooperativa Editorial Magisterio.

Inostroza, G., Jara, E. y Tagle, T. (2010). Perfil del mentor basado en competencias. Estudios Pedagogicos, 36(1), 117-129. DOI: https://doi. org/10.4067/S0718-07052010000100006

Jaeger, E. (2013). Teacher Reflection: Supports, Barriers, and Results. Issues in Teacher Education, 22(1), 89-104.

Lago, J. y Onrubia, J. (2008). Una estrategia general de asesoramiento para la mejora de la práctica educativa. Profesorado, 12(1), 1-13.

Lago, J. y Onrubia, J. (2011a). Asesoramiento psicopedagógico y mejora de la práctica educativa. Barcelona: Horsori.

Lago, J. y Onrubia, J. (2011b). Un modelo de asesoramiento para la mejora de las prácticas educativas. En E. Martín y J. Onrubia (Eds.), Proceso de innovación y mejora de la enseñanza (pp. 11-32). Barcelona: Graó.

LeCompte, M. D. \& Preissle, J. (1982). Problems of Reliability and Validity in Ethnographic Research. Review of Educational Research, 52(1), 31-60. DOI: https://doi.org/10.2307/1170272 
46 PRÁCTICAS DE MENTORÍA PARA LA INDUCCIÓN DE DOCENTES PRINCIPIANTES: ANÁLISIS DE CUATRO CASOS CHILENOS AL INICIO DEL PROCESO - S. Gorichon, M. Salas, M. Araos, M. Yáñez, A. Rojas-Murphy, G. Jara-Chandía

Lipton, L. \& Wellman, B. (2005). Mentoring matters: A practical guide to learning-focused relationships. USA: MiraVía.

Malderez, A. \& Bodóczky, C. (1999). Mentor courses. Cambridge: Cambridge University Press.

Malderez, A. \& Wedell, M. (2007). Teaching teachers: processes and practices. Londres: Continuum International Publishing Group.

Marcelo, C. (2002). Los profesores como trabajadores del conocimiento: Certidumbres y desafíos para una formación a lo largo de la vida. Educar, (30), 27-56.

Marcelo, C. (2009). Los comienzos en la docencia: un profesorado con buenos principios. Profesorado: Revista de Curriculum y Formación Del Profesorado, 1.

Melero, D. (2017). Mentoría para directores novatos en sistemas escolares del mundo. Centro de Desarrollo de Liderazgo Educativo. DOI: https://doi. org/10.1017/CBO9781107415324.004

Miguélez, M. (2006). Cualitativa, validez y confiabilidad en la metodología. Paradigma, 27(2), 7-33.

Ministerio de Educación de Chile, Mineduc. (2005). Informe Comisión sobre Formación Inicial docente. 1-94. Recuperado de http://www.oei.es/pdfs/ info_formacion_inicial_docente_chile.pdf

Ministerio de Educación de Chile, Mineduc. (2016). Ley No 20.903. Crea el sistema de desarrollo profesional docente y modifica otras normas.

Ministerio de Educación de Chile, Mineduc. (2018). Bases de postulación de docentes mentores al proceso de inducción y mentoría. Resolución exenta No 6627. Recuperado de https://www.cpeip.cl/wp-content/ uploads/2019/03/Bases-de-postulación-de-Mentores-Mentorías-2019. pdf

Moore-Russo, D. \& Wilsey, J. (2014). Delving into the meaning of productive reflection: A study of future teachers' reflections on representations of teaching. Teaching and Teacher Education, 37, 76-90.

Negrillo, C. e Iranzo, P. (2009). Formación para la inserción profesional del profesorado novel de educación infantil, educación primaria y educación secundaria: Hacia la reflexión desde la inducción y el soporte emocional. Profesorado, 13(1), 157-182.

Nuñez, C. G., Pino, M. y López, V. (2011). Horizontes de posibilidad de la mentoría en Chile: análisis de la experiencia piloto desde la perspectiva del discurso. Educação E Sociedade, 32(117), 1149-1164. 
Orland-Barak, L. (2010). Learning to mentor-as-praxis: Foundations for a curriculum in teacher education. New York: Springer.

Orland-Barak, L. (2016). Mentoring. In J. Loughran \& M. L. Hamilton (Eds.), International Handbook of Teacher Education (Vol. 2, pp. 105-141). DOI: https://doi.org/10.1007/978-981-10-0369-1_5

Orland-Barak, L. \& Klein, S. (2005). The expressed and the realized: mentors' representations of a mentoring conversation and its realization in practice. Teaching and Teacher Education, 21(4), 34-57.

Ortúzar, S., Flores, C., Milesi, C., Müller, M. y Ayala, P. (2011). Diseño de un programa de inducción en Chile para profesores en sus primeros años de ejercicio docente. En En Camino al Bicentenario. Concursos Políticas Públicas para Chile 2011 (pp. 267-305). Santiago de Chile: Ediciones Universidad Católica.

Pérez-Gómez, A. (coord.). (2010). Aprender a enseñar en la práctica: procesos de innovación y prácticas de formación en la educación secundaria. España: Graó.

Roca, B. (2018). Los recursos discursivos del asesor en un proceso de asesoramiento colaborativo (tesis doctoral). Universidad de Barcelona, Barcelona, España.

Roca, B. J., Armengol, M. À., Huerta, L. y Onrubia, J. (2019). Recursos discursivos del asesor en un proceso de asesoramiento colaborativo para la mejora de las prácticas docentes. Profesorado: Revista de Curriculum y Formación Del Profesorado, 23(2), 441.461. DOI: https:// doi.org/10.30827/profesorado.v23i2.9725

Rodríguez, E. (2007). Las instituciones también cuentan. Los profesores principiantes en educación media frente a los desafíos del desarrollo profesional. Docencia, 33, 64-73.

Ruffinelli, A. (2013). La calidad de la formación inicial docente en Chile: la perspectiva de los profesores principiantes. Calidad en la Educación, (39), 118. DOI: https://doi.org/10.31619/caledu.n39.80

Ruffinelli, A. (2014). Dificultades de la iniciación docente: iiguales para todos? Estudios Pedagogicos, 40(1), 229-242. DOI: https://doi.org/10.4067/ s0718-07052014000100014

Ruffinelli, A. (2020). Tutorías de práctica en la FID: condiciones, estrategias y efectos de la práctica reflexiva. Santiago de Chile.

Russell, T. (2012). Cambios paradigmáticos en la formación de profesores: peligros, trampas y la promesa no cumplida del profesional reflexivo. 
48 PRÁCTICAS DE MENTORÍA PARA LA INDUCCIÓN DE DOCENTES PRINCIPIANTES: ANÁLISIS DE CUATRO CASOS CHILENOS AL INICIO DEL PROCESO - S. Gorichon, M. Salas, M. Araos, M. Yáñez, A. Rojas-Murphy, G. Jara-Chandía

Encounters in Theory and History of Education, 13, 71-91. DOI: https:// doi.org/10.24908/eoe-ese-rse.v13i0.4426

Sanjurjo, L. (2012). Socializar experiencias de formación en prácticas profesionales: un modo de desarrollo profesional. Praxis Educativa, 16(1), 22-32.

Solís, M. C., Núñez, C., Vásquez, N., Contreras, I. y Ritterhaussen, S. (2016). Problemas en la inserción profesional de profesores: necesidad de mentoría. Estudios Pedagógicos (Valdivia), 42(4), 201-221. DOI: https:// doi.org/10.4067/S0718-07052016000500012

Tardif, M. (2004). Los saberes del docente y su desarrollo profesional. Madrid: Narcea.

Thompson, J. (2007). The first year teacher's survival guide. San Francisco: Jossey-Bass.

Vaillant, D. y Marcelo, C. (2015). El ABC y D de la formación docente. In Revista Española de Orientación y Psicopedagogía. Madrid: Editorial Narcea.

Vonk, J. (1995). Conceptualizing novice teachers' professional development. A base for supervisory interventions. Paper presented at AERA, San Francisco.

Willig, C. (2013). Introducing qualitative research in psychology. Berkshire: Open University Press.

Yin, R. (1989). Case Study Research. Design and Methods. London: SAGE.

Recibido: 20/03/2020

Aceptado: 12/06/2020 